\title{
Rasgos biográficos y bibliografía de Joseph de Tonquédec. Apuntes para la discusión sobre la inmanencia blondeliana
}

\author{
Luis Fernando Valdés \\ Universidad Panamericana
}

\begin{abstract}
French theologian Joseph de Tonquédec, sj, leads a large and heavy discussion about the notion of immanence, used by Maurice Blondel in order to explain his thought on the openness of the spirit to the supernatural. However, there is not much information about this theologian, well known by his opposition to Blondel. In this article, the Author presents a summary of that polemic, and then offers systematically and almost exhaustively the information about the life and works of Tonquédec, and also gives the bibliography about his position. All this material can be useful as documentary basis for placing the context, development and reception of the critical positions on blondelian immanence, in France, from 1912 to 1949.
\end{abstract}

La noción de inmanencia fue empleada por Maurice Blondel para dialogar con la filosofia moderna. Blondel pretendia mostrar que, lejos de encerrar al hombre en una autonomia, la inmanencia refleja la necesidad que el hombre tiene de abrirse a la trascendencia. Pero esta noción blondeliana tuvo una desafortunada recepción, en la que Joseph de Tonquédec tuvo un papel protagónico, al dar lugar a una polémica muy áspera. Para entender la filosofia blondeliana de la acción como una doctrina de la apertura del espíritu a lo sobrenatural, hace falta entender bien la noción de inmanencia, que se fue matizando gracias a estas discusiones.

$\mathrm{Y}$, para conocer el origen $\mathrm{y}$ los matices de la inmanencia blondeliana, es necesario profundizar en las discusiones que empezaron a finales de 1912, con la intervención de Tonquédec. Sin 
embargo, aunque estas controversias tuvieron mucho eco especialmente en los ambientes neoescolásticos-, han sido poco estudiadas. Por eso, a pesar de la influencia que ejerció en su época, ya que marcó el rumbo de la interpretación neoescolástica sobre las obras de Blondel, se tiene muy poca información sobre el P. de Tonquédec.

Con motivo de nuestra investigación sobre la apertura a lo sobrenatural en L'Action (1893), nos enfrentamos a la tarea de rastrear los datos biográficos y bibliográficos de este autor neoescolástico. A continuación presentamos los resultados de esa investigación, precedidos de un breve resumen sobre la discusión en torno a la inmanencia blondeliana.

\section{La discusión en torno a la noción blondeliana de inmanencia}

Por su larga y continua controversia con el autor de L'Action, Tonquédec ha sido considerado por algunos como el autor que ha realizado "la crítica más virulenta" que se haya hecho a los escritos de Blondel. ${ }^{1}$ Sin embargo, en su momento, esa crítica fue calificada por otros, como la única capaz de mostrar los errores de la doctrina blondeliana.

1 Cf. Claude Troisfontaines, "Présentation du tome II", en Maurice Blondel: Euvres complètes, II: 1888-1913. La philosophie de l'action et la crise moderniste. Texte établi et présenté par Claude Troisfontaines, Paris: PUF 1997, pp. xxxivXxxv: La critique plus virulent qui ait jamais été faite de ses écrits [c'est] celle du P. de Tonquédec.

Sin embargo, en cuanto al tono hostil, hubo otro autor más insidioso: M.-M. Gorce, $\mathrm{OP}$, que escribió un artículo "qui dépase Schwalm, Tonquédec et Garrigou en incompréhension hostile" (M. BLONDEL: Carta a Wehrlé, 18-III-1935, en M. Blondel - J. WEHRLÉ: Correspondance, $I I$, Commentaires et notes par Henri de Lubac, Paris: Aubier-Montaigne 1969, p. 687). La carta hace referencia a M.-M. GORCE: "Le néo-réalisme bergsonien-thomiste", Sophia (Napoli) 3 (1935), pp. 35 48. Vid. et M. Blondel - A. Valensin, Correspondance, III: (1912-1947), Texte annoté par Henri de Lubac, Paris: Aubier-Montaigne 1965, pp. 190-3, especialmente p. 192 nota $90,2$. 
Las criticas del $\mathrm{P}$. de Tonquédec a Blondel se extendieron desde 1912 hasta 1950, unos meses después de la muerte del filósofo de Aix. Los puntos a los que se dirigía se pueden clasificar en dos grandes temas. Por un lado, el P. de Tonquédec censuró ampliamente la noción de inmanencia de Blondel. Por otra parte, refutó el valor que el autor de L'Action daba al conocimiento, aunque esta controversia tuvo menos eco.

El inicio de la polémica sobre la inmanencia entre Maurice Blondel y Joseph de Tonquédec ${ }^{2}$ se sitúa, temporalmente, en los años siguientes a la condena del modernismo, por parte del Papa Pío X (1907). ${ }^{3}$ Pero las ideas y motivos de esa crítica conectan con algo anterior: con las reacciones que siguieron a la publicación de la Lettre sur l'apologétique (1896), ${ }^{4}$ en la que Blondel justificaba su postura ante los profesores parisinos, que rechazaban el carácter filosófico de su obra L'Action (1893). ${ }^{5}$

2 Sobre este tema vid. L.F. VALDÉs: "La apertura del espiritu a lo sobrenatural. El planteamiento blondeliano y su recepción", Excerpta e dissertationibus in sacra theologia 42 (2002) (en imprenta).

${ }^{3}$ En 1907 tuvieron lugar las intervenciones pontificias a propósito del modernismo. El 3 de julio, el Santo Oficio promulgó el decreto Lamentabili sane exitu (Acta Sanctae Sedis 40 (1907), pp. 470-8), que condenaba 65 proposiciones de los modernistas. Más adelante, el 8 de septiembre de 1907, el Papa Pio X publicó lá Encíclica Pascendi dominici gregis (Acta Sanctae Sedis 40 (1907), pp. 593-650), en la que aparecía el término "modernismo" aplicado al movimiento intelectual que causaba tanta agitación. Finalmente, en 1910, el mismo Romano Pontifice publicó el "motu proprio" Sacrorum antistitum (Acta Apostolicae Sedis 2 (1910), pp. 655-80), que contenia el juramento anti-modernista.

${ }^{4} \mathrm{M}$. BLoNDEL: "Lettre sur les exigences de la pensée contemporaine en matière d'apologétique et sur la méthode de la philosophie dans l'étude du problème religieux", Annales De Philosophie Chrétienne 33 (1896), pp. 337-47; 467-82; 599616; 34 (1896), pp. 131-47; 255-6\% y 337-50. Reproducido en M. BLONDEL: Eruvres cumpletes, II..., pp. 101-73. Versión castellana: M. BLONDEL: Carta sobre las exigencias del pensamiento contemporáneo en materia de apologética y sobre el método de la filosofia en el estudio del problema religioso, Trad. J.M. 'sasi, Bilbao: Universidad de Deusto 1990.

${ }^{5} \mathrm{M}$. BLONDEL: L'Action. Essai d'une critique de la vie et d'une science de la pratique, Paris: Bibliothèque de Philosophie Contemporaine 1993, xxv $+496 \mathrm{pp}$. Reproducido en Eurres complètes, I..., pp. 15-530. Versión castellana: M. BLONDEL: La Acción, traducción, introducción y notas de J.M. Isasi y C. Izquierdo, Madrid: BAC 1996. 
L'Action se presenta como una justificación racional de la apertura del espíritu desde la inmanencia. ${ }^{6}$ Ahí Blondel busca reconciliar dos nociones opuestas -inmanencia y trascendencia-, tomando el sujeto como el punto de partida. Mediante el método de inmanencia, el fílósofo de Aix realizá un estudio fenomenológico de la voluntad. La voluntad quiere "algo", un objeto que pueda satisfacer su impulso infinito, y de este modo, el sujeto pueda encontrar el : sentido de su vida. La voluntad presenta una dialéctica en la que los actos concretos de la voluntad (volonté voulue) no son suficièntes para saciar el deseo infinito que presenta el sujeto (volonté voulant). Là respuesta a esta "ecuación" entre la voluntad querida y la voluntad que quiere apunta a que el espíritu humano está abierto a la trascendencia, hacia "algo" infinito; capaz de resolver satisfacer el impulso de la voluntad. Pero esa apertura no demuestra la existencia de lo sobrenatural, sino sólo manifiesta la necesidad que nosotros tenemos de él. ${ }^{7}$

En la Lettre, Blondel intentó defender su postura ante el mundo académico francés, ${ }^{8}$ justificando la validez tanto de su noción como

${ }^{6}$ Cf. "La filosotĩa de la inmanencia en Fides et Ratio, 59: Una alusión a Maurice Blondel", Tópicos 19 (2000), pp. 211-31: 217-21.

${ }^{7}$ Más aún, la voluntad toma lo sobrenatural como una hipótesis, con el fin de afirmar desde la inmanencia la "necesidad para nosotros" de adherimos a lo sobrenatural (cf. C. IZQUIERDO: "La necesidad para nosotros de adherimos a lo sobrenatural", Scripta Theologica 31 (1999), pp. 531-40).

"La argumentación blondeliana tiene como objetivo afirmar filosóficamente lo sobrenatural 'en nosotros'. No se trata, pues, de demostrar la existencia de lo sobrenatural, sino de mostrar que lo sobrenatural tiene una huella en lo natural, que se manifiesta como una 'necesidad' en el hombre, aunque inalcanzable mediante las fuerzas humanas. La afirmación inmanente de esta necesidad para nosotros de lo sobrenatural es propiamente la apertura. Por eso, podemos decir que la doctrina blondeliana no es una filosofĩa de lo sobrenatural strictu sensu, sino de la apertura desde la inmanencia, es decir, del reflejo de lo sobrenatural en nosotros" (L.F. VALDÉS: La apertura del espiritu a lo sobrenatural. El planteamiento blondeliano y su recepción, Tesis doctoral, pro manuscripto, Pamplona: Universidad de Navarra 2000, pp. 111-12).

${ }^{8}$ El ambiente universitario francés de finales del s. XIX estaba dominado por el racionalismo."Blondel se encontraba ante un panorama académico que no tomaba en cuenta la religión. La influencia de la filosofia de Kant habia sido grande. Sobre la situación del kantismo y su relación con la teología a principios del s. XX, vid. E. COLOMER: El pensamiento alemán de Kant a Heidegger, I, Barcelona: Herder 1986, 
de su método de inmanencia. Aunque no pretendia polemizar con los autores neoescolásticos, la imprecisión terminológica respecto al tomismo y su combativo estilo literario le condujeron a una larga polémica con autores tomistas.

Tonquédec publicó una serie de artículos que luego darian lugar a su conocido libro Immanence, ${ }^{9}$ en el que presentaba una crítica a la filosofia de L'Action. El teólogo francés negaba la distinción empleada por Blondel en la Lettre, entre método de inmanencia y doctrina de inmanencia. Además interpretó la noción blondeliana de acción como un principio de interdependencia. Tonquédec identificaba la noción de inmanencia - a la que llamó "principio de inmanencia"-, con ese principio de interdependencia. Según este principio, todo se apoya en todo, por una conexión inmanente, de modo que no se conoce nada sino hasta el final. Pero como nunca es posible conocer todo, se cae en el escepticismo. Consideraba que Blondel fue demasiado lejos al utilizar la noción de inmanencia, considerada como la condición de toda doctrina filosófica y, por eso, en la práctica, Blondel tendría que admitir un cierto inmanentismo.

Tonquédec analizó la filosofia blondeliana desde una visión intelectualista, pues consideraba que el intelectualismo es el tomismo más puro, y que ese tomismo era la doctrina oficial de la Iglesia. Según esta visión, el conocimiento de Dios debe ser afirmado desde el pensamiento, pero Blondel utiliza otro medio, que es la "sola acción". Esa sola acción se opone al pensamiento.

El teólogo francés reconocía que, en su demostración de la necesidad de Dios, Blondel no realizó ninguna afirmación que lo

327 pp; H. KUHN: "La filosofia del espiritu y los idealismos recientes", en $\mathrm{H}$. VORGRIMLER (dir.): La teologia del siglo XX. Perspectivas, corrientes y motivaciones en el mundo cristiano y no cristiano, I, Madrid: BAC 1973, pp. 23140; I. Mancini: Kant e la teologia, Assisi: Cittadella 1975, 250 pp.; E. Vilanova: Historia de la teología cristiana, III, Barcelona: Herder 1992; Ch. WILD: "La herencia de Kant y del idealismo alemán", en H. VORGRIMLER (dir.), La teologia..., pp. $241-48$.

9 J. DE TONQUÉDEC: Immanence. Essai critique sur la pensée de M. Blondel, Paris: Beauchesne 1913, xv-307 pp. En 1932 se publicó la tercera edición con una introducción ampliada: cxi-316 pp. 
hiciera caer ni en el inmanentismo ni en el idealismo. Pero lo consideraba cercano a esas posturas, pues el jesuita no admitía que fuera epistemológicamente posible distinguir entre el método de inmanencia y la doctrina de inmanencia, porque consideraba que todo método siempre estaría solidarizado con una doctrina.

Esta interpretación de la doctrina blondeliana desde categoría del pensaniento tradicional influyó en autores importantes. En-1913, Reginald Garrigou-Lagrange se hizo eco de Immanence. En la Revue Thomiste, apoyó con un par de artículos las criticas que Tonquédec dirigió a Blondel. ${ }^{10}$

\section{Apuntes biográficos de Joseph de Tonquédec}

¿Quién era Joseph de Tonquédec? Era un bretón nacido en Molaix (Finistère), ${ }^{11}$ el 27 de octubre de 1869..2 Ingresó ẹn la Compañia de Jesús y después fue profesor de filosofia en París. Durante varios años fue redactor de la revista Études. Falleció en Paris, el 22 de noviembre de $1962 .^{13}$

Louis Jugnet, discipulo suyo, lo describia como un hombre de talla mediana, cuyo rostro "parecía esculpido en piedra", de ojos claros y de voz fuerte y clara; usaba una barba que le daba. un aire de

${ }^{10}$ R. Garrigou-Lagrange: "Autour du blondélisme et du bergsonnisme", Revue thomiste 21 (1913), pp. 350-77: 351-71; R. GARRIGOU-LAGRANGE: "Les méthodes de l'Apologétique", Revue thomiste 21 (1913), pp. 481-85: 471-85.

11 Sobre la vida y las obras de Tonquédec vid. L. JugnET: "Traditionnel et moderne: le R.P. de Tonquédec", La Pensée Catholique 84 (1963), pp. 24-43; C. WENIN: sección "Chronique. France", Revue Philosophique 61 (1963), p. 156; A. DEL TORO: "Neotomismo y filosofia y teología en el siglo XX", en Gran Enciclopedia Rialp, XVI, Madrid 61989, p. 739 (a partir de aquí, citaremos por GER con los números de tomo y de página); A. DEL TORO, "Realismo II, 3. Neoescolásticos", en GER XIX, 727, noticia necrológica en Tidschift voor Philosophie 24 (1962), p. 785.

12 Empleamos los datos proporcionados por Jugnet, ya que fue su discípulo desde el curso 1933-34 hasta su muerte. Según Wenin y del Toro, Tonquédec nació en 1868.

13 Jugnet, quien estuvo en contacto con Tonquédec en sus últimos tiempos ("derniers temps", sin especificar más), afirma que falleció el 22-XI-1962. Wenin fija la fecha un día antes: el 21-XI-1962. Del Toro, en GER:XVI, 739 pone la fecha de deceso en 1959; pero en su otro artículo, en GER XIX, 727, afirma que murió en 1962. 
"capellán de la marina". El P. de Tonquédec era estricto —“más valía no hacerlo enojar"-; la reserva de sus sentimientos podía hacerlo parecer como insensible, pero bajo las apariencias un poco bruscas sabía tener "muestras de afecto, incluso de ternura" hacia los que convivían con él. ${ }^{14}$

El P. de Tonquédec era un hombre piadoso, con una profunda devoción a la Virgen María. Era un director espiritual con sentido común y fortaleza. Durante veinte años desempeñó el cargo de exorcista oficial de la diócesis de París. ${ }^{15}$

El jesuita era un hombre culto que sabia -y escribía- de filosofia, teologia, psiquiatria, ${ }^{16}$ actualidad política ${ }^{17}$ y literatura.

14 Cf. L. JUGNET: "Traditionnel et moderne...", pp. 24-5.
15 Cf. A. GRÉGOIRE, sj: recensión de Les maladies nerveuses ou mentales et les manifestations diaboliques, Nouvelle Revue Théologique 2 (1939), p. 238.

16 "La formation psychiatrique du Père de Tonquédec était solide, mais fondamentalement tributaire de la pensée française classique, un peu courte á notre gré par rapport aux travaux psychopathologiques des Allemands, des Autrichiens et des Suisses. Conquis un moment par la distinction entre 'méthode psychanalytique' et 'doctrine freudienne', à quelques réserves près, le Père devait plus tard abandonner celle-ci pour l'essentiel (...)" (L. JUGNET: "Traditionnel et moderne...", p. 41 , nota 54).

17 Jugnet afirma que Tonquédec "il aima et admira de tout cour Maurras, qui le lui rendit bien" (L. JUGNET: “Traditionnel et moderne...", p. 33).

Fundada en 1899 por Henri Veugeois, l'Action Française fue un movimiento intelectual y politico, de corte nacionalista, monárquico y antidemocrático. Sus órganos de expresión eran la Revue d'Action Française y el diario L'Action Française. Más tarde fue dirigido por Charles Maurras, escritor prolifico y polemista brillante, y por León Daudet. Maurras, discípulo de Comte, mantenía la primacía de lo social sobre lo individual e identiticaba lo social con las naciones. Aunque se confesaba agnóstico, Mauras veia en la religión católica, por la lógica y solidez de su organización, una de la piezas fundamentales de su estrategia política. Durante la política anticatólica del Presidente Combes, muchos grupos católicos se pusieron bajo el liderazgo de Maurras, quien corrigió sus expresiones hacia la Iglesia y se hizo asesorar por teólogos.

Debido a frecuentes denuncias, Pío X encargó al Santo Oficio un dictamen sobre la obra de Maumas. El Dicasterio redactó un documento (26-I-1914) que prohibía siete libros de Maurras y la revista, pero no el periódico. Tanto Pío X como Benedicto $\mathrm{XV}$ retuvieron el escrito. En cambio, Pío XI revisó personalmente el caso, y el 26XII-1926 hizo publicar el decreto del Santo Oficio de 1914, ratificándolo (se ponían en el Index los siete libros de Maurras y la revista) y condenando el periódico. (Cf. 
Además, era un "poliglota destacado". ${ }^{18}$ Entre su grupo de discípulos y amigos era conocido como un "defensor constante de la verdad", como uno de los "pensadores más seguros" en cuanto a la doctrina.

Tonquédec "siempre subrayó la importancia capital de los estudios filosóficos bien hechos, y la nocividad irremediable de la 'falsa filosofia' para la fe religiosa y el equilibrio humano, individual y colectivo".!9 Escribió mucho sobre filosofia, con un estilo clásico y a la vez muy claro, y con una gran contundencia lógica: una vez puestos los principios, iba directamente a las consecuencias. ${ }^{20}$

Su actitud intelectual intentó ser la de un fiel discipulo de santo Tomás de Aquino. Toda su obra fue escrita bajo esta inspiración: Su objetivo era presentar "el tomismo integral, orgánico, tal como santo Tomás lo ha concebido". ${ }^{21}$ Jugnet resume el diálogo de Tonquédec con la filosofia moderna, con la siguiente expresión: "jconocer [la filosofia moderna] no es aceptar[la], comprender[la] no es aprobar[la]!".22 Sin embargo, no todos están de acuerdo con que haya logrado esá armonía. ${ }^{23}$

El principio central de su filosofia fue el intelectualismo, ${ }^{24}$ en el que apoyaba su fidelidad a la doctrina del Aquinate. Creía firmemente que el tomismo podía perfectamente, e incluso debia, sèr calificado de "realismo crítico". Sería "crítico" no por amalgamarse

J. Escudero ImberT: "El dificil pontificado de Pío XI", en J.I. Saranyana (ed.): Cien años de pontificado romano. De León XIII a Juan Pablo II, Pamplona: Eunsa 1997, pp. 100-2, y R. GUTIÉRREZ NIETO: "Action Française", en GER I, 157-8).

18 L. JUGNET: "Traditionnel et moderne...", p. 25; entre otras lenguas, Tonquédec "lisait excellemment l'allemand". (cf. L. JugneT: "Traditionnel et moderne...", p. 37).

${ }^{19}$ L. JUGNET: "Traditionnel et moderne...", p. 26.

${ }^{20} \mathrm{Cf}$. E. BRISBoIs, sj: La critique de la connaissance (recensión), Nouvelle Revue Théologique 7(1930), p. 600 .

${ }^{21} \mathrm{~J}$. DE TONQUÉDEC: Les principes de la Philosophie thomiste. La critique de la connaissance, Paris: Bibliothèque des Archives de Philosophie, Beauchesne 1929, p. XVII.

${ }^{22}$ L. JuGNET: "Traditionnel et moderne...", p. 33.

${ }^{23}$ Cf. E. BRISBOIS, sj: La critique...

${ }^{24}$ C. WENIN: sección "Chronique..." 
con el cartesianismo ni con el kantismo, sino porque analizaría reflexivamente y justificaría la noción realista del conocimiento. ${ }^{25}$ Sin embargo, se le criticó que en su obra La critique de la connaissance, no hubiera expuesto lo que se entiende, después de Kant, por problema crítico, y que no hubiera dicho de qué manera, en virtud de sus principios, el sistema filosófico de santo Tomás debía reaccionar ante un problema de este tipo. ${ }^{26}$

Esta postura gnoseológica llevó a Tonquédec a enfrentarse primero a Bergson y luego a Blondel. La estrategia dialéctica del jesuita consistia en elaborar una síntesis doctrinal a partir de los escritos de su rival de turno. Esa síntesis, estructurada según el canon escolástico, recogería literalmente las principales tesis de sus adversarios. Luego, contrastaba esas tesis con la doctrina tomista; y después, acercaba esas tesis a las posturas ya condenadas por el Magisterio. En general esas síntesis tomaban en cuenta frases comprometedoras, pero prescindian del contexto argumentativo, de donde eran tomadas.

Este método fue la causa de los malentendidos y las continuas polémicas. Se le criticó, por ejemplo, de que a pesar de sus esfuerzos por penetrar la filosofia bergsoniana, "las observaciones del Rev. Padre, apoyadas por el texto y la letra, no alcanzan siempre

\footnotetext{
25 L. Jugnet: "Traditionnel et moderne...", p. 28.

${ }^{26} \mathrm{Cf}$. E. Brisbols, sj: La critique...: "Dans tout son exposé, le P. de T. entende bien ne nous donner que la pensée de saint Thomas, rien que le pur thomisme, celui de saint Thomas et pas un autre. Et il faut le reconnaitre, ce qu'il nous donne comme étant la. pensée du maître est du thomisme bien authentique. Mais l'ensemble de l'auvre constitue-t-elle vraiment la critique de la connaissance d'après saint Thomas? L'auteur lui-même le reconnaît, nous n'avons nulle part, dans l'auvre de l'Aquinate, quelque chose que ressemble à une critique de la connaissance telle que l'entend la philosophie moderne. On se serait attendu tout au moins dans un ouvrage tel que le sien, intitulé: La critique de la connaissance, à ce que l'auteur nous ê̂t exposé ce que l'on entend, depuis Kant, par le problème critique, et nous eût dit comment, en vertu de ses principes, le système philosophique de saint Thomas devait réagir devant le problème ainsi posé".
} 
perfectamente el espiritu que las inspira". ${ }^{27}$ Blondel le dirigiría la misma queja más adelante. ${ }^{28}$

\section{Obras de Joseph de Tonquédec}

En este apartado exponemos el elenco de las obras escritas por Tonquédec. Las agrupamos según su temática: sobre Blondel, sobre apologética, sobre filosofia clásica, sobre Bergson, sobre teología, filosofia y literatura modernas y sobre política, espiritualidad y psicología religiosa. Cada sección de este apartado está ordenada cronológicamente, salvo cuando se indica que se emplea otro criterio.

a. Sobre M. Blondel

J. de TONQUÉDEC:

- "L'idée d'immanence chez M. Blondel", en Revue practique d'Apologétique 15 (1 déc. 1912) 347-361 y (15 déc. 1912) 419-435.

— "L'Action réalisatrice", en Études 133 (20 déc. 1912) 766-780.

- "Observations' du P. de Tonquédec", en Revue practique d'Apologétique 15 (15 janv. 1913) 593-599. Reproducido en Annales De Philosophie Chrétienne 165 (févr. 1913) 551-556, y en M. BLONDEL, CEuvres complètes, II: 1888-1913. La philosophie de l'action et la crise moderniste. Texte établi et présenté par Claude Troisfontaines, PUF, Paris 1997, pp. 763768.

- A propos d'une bruchure récente de M. Blondel, Beauchesne, Paris 1913, $15 \mathrm{p}$.

- Immanence. Essai critique sur la doctrine de M. Maurice Blondel, París 1913, xv-307 pp. La tercera edición aumentada se hizo en: Beauchesne, Paris 1932, cx-315 pp.

${ }^{27}$ G. DelanNoye, sj: Sur la Philosophie bergsonnienne (recensión), Nouvelle Revue Théologique 6 (1938), p. 753.

${ }^{28}$ M. Blondel: Carta a Valensin, 5-XII-1912, en M. Blondel - A. VAlENSIN: Correspondance, III..., pp. 28-9. 
- Recensión de: J. MARTAIN, Réflexions sur l'intelligence et sa vie propre, en Bulletin thomiste 29 (sept.-oct. 1924) 115-120. (Crítica a Le procés de l'intelligence).

- "Qu'est-ce que la Mystique?", en Revue d'Apologétique 43 (15 nov. 1926) 208-218.

- "La doctrine blondélienne de la connaissance d'après La Pensée", en Revue de Philosophie (1935) 481-512.

- Deux études sur "La Pensée" de M. Maurice Blondel, Beauchesne, Paris 1936, 177 pp.

- "Les premières principes de la raison dans "L'Être et les êtres" de M. Blondel", en Revue de Philosophie, 36 (sept.-oct. 1936) 381-400.

— "Pourquoi j'ai critiqué Maurice Blondel", en Revue Thomiste, 49 (juil.-sept. 1949) 563-580.

- "Maurice Blondel et la théologie", en Recherches de Science Religieuse 37 (1950) 98-105.

b. Sobre apologética en general

J. DE TONQUÉDEC:

- Introduction à l'étude du merveilleux et du miracle, Beauchesne, Paris 1916,.XVI-461 pp. $3^{\text {a }}$ ed. en 1923.

- "Milagro", en Dictionnaire Apologétique de la Foi Catholique, III, col. 517-578, Paris ${ }^{1} 1916$.

- "La critique de temoignage en matière de merveilleux", en Recherches de Science Religieuse 6 (1916) 50-112.

- "Immanence. I. Étude ", en Dictionnaire Apologétique de la Foi Catholique, II, col. 593-612, Paris ${ }^{2} 1926$.

— "La Spiritualité de la Théosophie", en L. DE GRANDMAISON - J. $\mathrm{DE}$ TONQUÉDEC, La théosophie e l'anthroposophie, Beauchesne, Paris 1939, 192 pp. Este articulo ya habia aparecido en Études (déc 1938).

- Merveilleux métaphysique et miracle chrétien. Coll. Centre d'études Laënnec. Lethielleux, Paris 1955, 134 pp. 
c. Sobre filosofia clásica

\section{J. de TONQUÉDEC:}

- Les principes de la Philosophie thomiste. La critique de la connaissance, Bibliothèque des Archives de Philosophie, Beauchesne, Paris 1929, xxx-568 pp. La tercera edición se hizo en: Coll. Les principes de la Philosophie thomiste, I. Lethielleux, Paris 1961, xxx-568 pp.

- Questions de cosmologie et de physique chez Aristote et Saint Thomas, Vrin, Paris 1950, 127 pp.

- La Philosophie de lä nature. Première partie: la nature en général. Prolégomènes, Coll. Les principes de la Philosophie thomiste, II, Lethielleux, Paris (3 fasc. 1956, 1958, 1959).

- La Philosophie de la nature. Deuxième partie: la nature vivante et connaissante, Coll. Les principes de la Philosophie thomiste, II, Lethielleux, Paris 1963, 124 pp.

— "De la qualité", en Revue de Metaphysique et Morale 65 (1965) 246-252.

\section{d. Sobre H. Bergson}

J. de TONQUÉDEC:

- La notion de vérité dans la "Philosophie nouvelle", Beauchesne, Paris 1908, 152 pp. (Colección de artículos publicados en Études durante 1907).

- Dieu dans "L'évolution créatrice". Avec deux lettres de $M$. Bergson, Beauchesne, Paris 1912, 35 pp. Se trata de dos artículos publicados en Etudes (1908), precedidos de una breve introducción.

- Comment interpréter l'ordre du monde? A propos du dernier ouvrage de M. Bergson, Paris 1918.

- Une preuve facile de l'existence de. Dieu: l'ordre du monde, Beauchesne, Paris 1918, $30 \mathrm{p}$. 
- Sur la Philosophie bergsonnienne, Beauchesne, Paris 1936, 244 pp. Recopilación de escritos anteriores:

- Dieu dans "L'évolution créatrice". Avec deux lettres de M. Bergson, Beauchesne, Paris 1912.

- "Bergsonisme et Scolastique", en la Revue critique des idées et des lettres (déc. 1913).

- "La clef des "deux sources" de la morale et de la religion", en Études 213 (1932) 516-543.

- "Le contenu des deux sources", en Études (mars et avril 1933) 667-683.

- "La conception bergsonienne de Dieu", en AA. VV. Actes $d u X^{i m e}$ Congrès des Société philosophiques de langue française, Bulletin de la Société française de Philosophie, $n^{\circ}$ spécial, 1959.

e. Sobre teología, filosofia y literatura modernas

J. DE TONQUÉDEC, L'ceuvre de Paul Claudel, Beauchesne, Paris 1917. ( $3^{\mathrm{a}}$ ed. en 1927$)$.

- G. H. Chesterton: ses idées et son caractère, Beauchesne, Paris 1920.

- Une Philosophie existentielle. L'existence d'après Karl Jaspers, Bibliothèque des Archives de Philosophie. Beauchesne, Paris $1945, \mathrm{x}-142 \mathrm{pp}$.

f. Sobre politica, espiritualidad y psicología religiosa

Esta sección está ordenada por temas.

J. de TONQUÉDEC:

- "Les sciences sociales sont-elles des sciences morales?", en Nouvelles de Chrétienté 118 (15 mars 1957).

- "Ce que je sais de Dieu", en AA. VV., Ce que je sais de Dieu, Les cahiers contemporains, Paris 1926, pp. 11-18. 
- "Faut-il revenir au "thomisme"", en AA. VV., La renaissance religieuse, Bibliothèque de philosophie contemporain, Paris 1928 , pp. 145-163.

- Voilà votre Mère, Beauchesne, Paris. Reedición en Lethielleux, Paris $1950,38 \mathrm{p}$.

- La Vierge Marie, idéal et mère de la pureté, Beauchesne, Paris.

- Marie Mère de Dieu, Beauchesne, Paris.

- Les maladies nerveuses ou mentales et les manifestations diaboliques, Beauchesne, Paris 1938, 242 pp. Trad. castellana: ¿Acción diabólica o enfermedad?; 1948.

- Voz "Apparitions", en Dictionnaire de Spiritualité, fasc. III, col. 801-809.

- Voz "Sanctification des anormaux", en Dictionnaire de Spiritualité.

\section{Obras sobre Joseph de Tonquédec}

En este apartado presentamos el listado de la bibliografia crítica sobre Tonquédec. Las secciones siguen prácticamente el mismo orden que las secciones del apartado anterior sobre las obras de Tonquédec. De este modo se facilitará encontrar las reacciones que suscitaron sus escritos. Las recensiones sin firma aparecen inmediatamente después de los artículos firmados, y siguen un criterio cronológico.

\section{a. Vida y obras de J. de Tonquédec}

L. JUGNET, "Traditionnel et moderne: le R. P. de Tonquédec", en Pensée Catholique 84 (1963) 24-43.

A. DEL TORO, "Neotomismo y filosofia y teología en el siglo XX", en GER 16, Madrid ${ }^{6} 1989$, p. 739.

A. DEL TORO, "Realismo II, 3. Neoescolásticos", en GER 19, Madrid ${ }^{6} 1989$, p. 727.

C. WENIN, sección "Chronique. France", en Revue Philosophique 61 (1963) 156. 
Noticia necrológica sin firma, en Tidschift voor Philosophie 24 (1962) 785.

\section{b. Sobre las polémicas de Tonquédec con Blondel}

H. BOUILLARD, "L'intention fondamentale de Maurice Blondel et la théologie", en Recherches de Science Religieuse 36 (1949) 321-402.

- "Maurice Blondel et la théologie (Réponse au P. de Tonquédec)", en Recherches de Science Religieuse 37 (1950) 105-1 12.

G. COLOMBO, Del Soprannaturale, Glosa, Milano 1996.

G. DE BROGLIE, recensión de: J. DE TONQUÉDEC, Deux études sur "La Pensée" de M. Maurice Blondel, en Nouvelle Revue Théologique, 64 (avr. 1937) 342.

P.-M. CONTERSON, OP, "Surnaturel", en Bulletin Thomiste 8 (1953) 800-801. (Recensión de: J. DE TONQUÉDEC, "Maurice Blondel et la théologie", y H. BOUILLARD, "L'intention fondamentale de Maurice Blondel et la théologie").

H. DELAHAYE, recensión de: J. DE TONQUÉDEC, Immanence ( $3^{\mathrm{a}}$ ed.), en Revue Universelle 56 (1 déc. 1933) 627-628.

G. F. recensión de: J. DE TONQUÉDEC, "La doctrine blondélienne de la connaissance d'après La Pensée", en Divus Thomas 39 (1936) 287-288.

- Recensión de: J. DE TONQUÉDEC, Deux études sur "La Pensée" de M. Maurice Blondel, en Divus Thomas 39 (1936) 554-555.

R. GARRIGOU-LAGRANGE, Autour du blondélisme et du bergsonnisme, en Revue thomiste 21 (mai-juin 1913) 350-377.

- "Les méthodes de l'Apologétique", en Revue thomiste 21 (1913) 481-485.

C. GIRY, recensión de: J. DE TONQUÉDEC, Immanence, en La croix (19 juin 1913).

R. JOLIVET, recensión de: J. DE TONQUÉDEC, "Deux études sur $L a$ Pensée de M. Maurice Blondel", en Revue apologétique 63 (févr. 1937) 221-222. 
L. MAISONNEUVE, recensión de: J. DE TONQUÉDEC, Immanerice, en Bulletin de Littérature ecclésiastique 15 (15 avr. 1913) 18890.

J. MESSAUT, recensión de: J. DE TONQUÉDEC, "Deux études sur La Pensée de M. Maurice Blondel", en Revue thomiste 41 (1936) 670.

- Recensión de: J. DE TONQUÉDEC, Immanence ( $3^{\mathrm{a}} \mathrm{ed}$.), en Revue thomiste 39 (1934) 441.

A. MICHEL, recensión de: J. DE TONQUÉDEC, Immanence ( $3^{\mathrm{a}}$ ed.), en Bulletin Thomiste 4 (1934) 324.

- Recensión de: J. DE TONQUÉDEC, "Pourquoi j'ai critiqué Maurice Blondel", en L'Ami du Clergé 61 (27 sept. 1951) 583-584.

F. PRADEL, Autour de la méthode d'immanence, en Revue de Philosophie 22 (févr. 1913) 286-303. (Recesión de Immanence).

Albert VALENSIN, recensión de: J. DE TONQUEDEC, Immanence, en Université catholique 73 (15 juin 1913) 175-180. Respuesta de Tonquédec en Université catholique 73 (15 août 1913) 356-357: Contrarréplica de Valensin en Université catholique (15 août 1913) 358-361:

Recensión sin firma sobre J. DE TONQUÉDEC, "L'action réalisatrice", en Annales De Philosophie Chrétienne 165 (janv. 1913) 435438 .

Recensión sin firma sobre J. DE TONQUÉDEC, Immanence, en L'Ami du Clergé, 35 (7 août 1913) 681-682.

Recensión sin firma sobre J. DE TONQUÉDEC, Immanence ( $3^{\mathrm{a}} \mathrm{ed}$.), en L'Ami du Clergé 50 (21 sept 1933) 606.

Recensión sin firma sobre J. DE TONQUÉDEC, "Deux études sur La Pensée de M. Maurice Blondel", en Revue de Metaphysique et Morale 44 (suppl. janv. 1937) 6-7. 
c. Sobre las obras apologéticas de Tonquédec

R. CATHALA, recensión de: J. DE TONQUÉDEC, Introduction à l'étude du merveilleux et du miracle, en Revue thomiste 24 (1919) 295-296.

J. GALOT, S.J., recensión de: J. DE TONQUÉDEC, Merveilleux métaphysique et miracle chrétien, en Nouvelle Revue Théologique 2 (1957) 312.

H. D. GARDEIL, "Dieu", en Bulletin Thomiste 8 (1953) 840-841. (Recensión de: J. DE TONQUÉDEC, Une preuve facile de l'existence de Dieu: l'ordre du monde).

M. LABOURDETTE, recensión de: J. DE TONQUÉDEC, Merveilleux métaphysique et miracle chrétien, en Revue thomiste 61 (1961) 630-631.

A. MICHEL, recensión de: J. DE TONQUÉDEC, "La clef des "deux sources" de la morale et de la religion", en Bulletin Thomiste 4 (1934) 325 .

P. M., recensión de: J. DE TONQUÉDEC, Merveilleux métaphysique et miracle chrétien, en Razón y fe 154 (1956) 485.

F. TAYMANS, S.J., recensión de: L. DE GRANDMAISON - J. DE TONQUÉDEC, La théosophie e l'anthroposophie, en Nouvelle Revue Théologique 8 (1939) 1014-1015.

d. Sobre las obras de filosofia clásica de Tonquédec

A. D'ALÉS, recensión de: J. DE TONQUÉDEC, La critique de la connaissance, en Recherches de Science Religieuse 19 (1929) 545 .

E. BRISBOIS, S. J., recensión de: J. DE TONQUÉDEC, La critique de la connaissance, en Nouvelle Revue Théologique 7 (1930) 600.

S. D., recensión de: J. DE TONQUÉDEC, La critique de la connaissance $\left(3^{\mathrm{a}}\right.$ ed.), en Nouvelle Revue Théologique 4 (1965) 442-443. 
L. P. DELGADO, recensión de: J. DE TONQUÉDEC, La Philosophie de la nature. Deuxième partie: la nature vivante et connaissante, en Razón y fe 165 (1962) 324.

É. DES PLACES, recensión de: J. DE TONQUÉDEC, Questions de cosmologie et de physique chez Aristote et Saint Thomas, en Recherches de Science Religieuse 37 (1950) 317.

D. DOMÍNGUEZ, recensión de: J. DE TONQUÉDEC, Questions de cosmologie et de physique chez Aristote et Saint Thomas, en Pensamiento 7 (1951) 270-271.

H. D. GARDEIL, recensión de: J. DE TONQUÉDEC, La Philosophie de la nature. Première partie: la nature en général, fasc. 1, en Bulletin Thomiste 10 (1957) 281-282.

J. GLBERT, recensión de: J. DE TONQUÉDEC, La Philosophie de la nature. Première partie: la nature en général, en Nouvelle Revue Théologique 10 (1958) 1123.

E. GLSON, Réalisme thomiste et critique de la connaissance, Vrin, Paris 1939.

JOURNET, recensión de: J. DE TONQUÉDEC, La Philosophie de la nature. Première partie: la nature en général, fasc. 2-3, en Nova et. Vetera 34 (1959) 155-156.

V. LE:OY, recensión de: J. DE TONQUÉDEC, La critique de la connaissance ( $3^{\mathrm{a}}$ ed.), en Revue thomiste 62 (1962) 168-169.

- Recensión de: J. DE TONQUÉDEC, La Philosophie de la nature. Première et deuxième partie, en Revue thomiste 67 (1963) 649-653.

B. MONTAGNES, recensión de: J. DE TONQUÉDEC, La critique de la connaissance ( $3^{\mathrm{a}}$ ed.), en Revue des Sciences philosophiques et théologiques 46 (1962) 721.

L. PRIETTO, recensión de: J. DE TONQUÉDEC, La Philosophie de la nature. Première partie: la nature en général, en Razón y fe 163 (1961) 653.

H.-D. SIMONIN, O. P., recensión de: J. DE TONQUÉDEC, La critique de la connaissance, en Bulletin Thomiste 3 (1930) 1-16. 
- Recensión de: J. DE TONQUÉDEC, Questions de cosmologie et de physique chez Aristote et Saint Thomas, en Bulletin Thomiste 8 (1951) 233-234.

- Recensión sin firma sobre J. DE TONQUÉDEC, La Philosophie de la nature. Première partie: la nature en général, fasc. 1 , en Estudios Filosóficos 6 (1957) 530-531.

Recensión sin firma sobre J. DE TONQUÉDEC, La Philosophie de la nature. Première partie: la nature en général, fasc. 1, en Sciences Ecclésiastiques 9 (1957) 331.

Recensión sin firma sobre J. DE TONQUÉDEC, La Philosophie de la nature. Première partie: la nature en général, fasc. 1 , en Études 295 (1957) 304.

Recensión sin firma sobre J. DE TONQUÉDEC, La Philosophie de la nature. Première partie: la nature en général, fasc. 1 , en Bijdragen. Tijdscrift voor philosophie en theologie 19 (1958) 112.

Recensión sin firma sobre J. DE TONQUÉDEC, La Philosophie de la nature. Première partie: la nature en général, fasc. 1, en Ephemerides.theologico lovanienses 34 (1958) 92.

Recensión sin firma sobre J. DE TONQUÉDEC, La Philosophie de la nature. Première partie: la nature en général, fasc. 1, en Salmanticensis 5 (1958) 267-268.

Recensión sin firma sobre J. DE TONQUÉDEC, La Philosophie de la nature. Première partie: la nature en général, fasc. 2-3, en Divus Thomas 62 (1959) 223.

Recensión sin firma sobre J. DE TONQUÉDEC, La Philosophie de la nature. Première partie: la nature en général, fasc. 2-3, en Pensamiento 15 (1959) 640-641.

Recensión sin firma sobre J. DE TONQUÉDEC, La Philosophie de la nature. Première partie: la nature en général, fasc. 2-3, en Lumière et Vie 8 (1959) 152.

Recensión sin firma sobre J. DE TONQUÉDEC, La Philosophie de la nature. Première partie: la nature en général, fasc. 2-3, en $L a$ Ciencia Tomista 86 (1959) 658-662. 
Recensión sin firma sobre J. DE TONQUÉDEC, La Philosophie de la nature. Première partie: la nature en général, fasc. 2-3, en Salmanticensis 6 (1959) 80.

Recensión sin firma sobre J. DE TONQUÉDEC, La Philosophie de la nature. Première partie: la nature en général, fasc. 2-3, en La Ciudad de Dios 172 (1959) 385.

Recensión sin firma sobre J. DE TONQUÉDEC, La Philosophie de la nature. Première partie: la nature en général, fásc. 2-3, en Études 302 (1959/3) 138.

Recensión sin firma sobre J. DE TONQUÉDEC, La Philosophie de la nature. Première partie: la nature en général, fasc. 2-3, en Revista de Filosofia 18 (1959) 516-517.

Recensión sin firma sobre J. DE TONQUÉDEC, La Philosophie de la nature. Première partie: la nature en général, fasc. 2-3, en Bijdragen. Tijdscrift voor philosophie en theologie 20 (1959) 221.

Recensión sin firma sobre J. DE TONQUÉDEC, La Philosophie de la nature. Première partie: la nature en général, fasc. 2-3, en Sciences Ecclésiastiques 11 (1959) 290.

Recensión sin firma sobre J. DE TONQUÉDEC, La Philosophie de la ñature. Première partie: la nature en général, fásc. 2-3, en L'Ami du Clergé 69 (1959) 60.

Recensión sin firma sobre J. DE TONQUÉDEC, La Philosophie de la nature. Première partie: la nature en général, fasc. 2-3, en Revue des Sciences philosophiques et théologiques 43 (1959) 399.

Recensión sin firma sobre J. DE TONQUÉDEC, La Philosophie de la nature. Première partie: la nature en général, fasc. 2-3, en Revue Philosophique 57 (1959) 277-280.

Recensión sin firma sobre J. DE TONQUÉDEC, La Philosophie de la nature. Première partie: la nature en général, fasc. 2-3, en Revista de Filosofia 18 (1959) 516; 19 (1960) 80-81.

Recensión sin firma sobre J. DE TONQUÉDEC, La Philosophie de la nature. Première partie: la nature en général, fasc. 2-3, en The Modern Schoolman 37 (1959-60) 65-66. 
Recensión sin firma sobre J. DE TONQUÉDEC, La Philosophie de la nature. Première partie: la nature en général, fasc. 2-3, en Sciences Ecclésiastiques 11 (1959) 290-292; 12 (1960) 446447.

Recensión sin firma sobre J. DE TONQUÉDEC, La Philosophie de la nature. Première partie: la nature en général, fasc. 2-3, en Revue d'Histoire et de Philosophie religieuses 40 (1960) 9899.

Recensión sin firma sobre J. DE TONQUÉDEC, La Philosophie de la nature. Première partie: la nature en général, fasc. 2-3, en Études Philosophiques 14 (1959) 104-105; 15 (1960) 304.

Recensión sin firma sobre J. DE TONQUÉDEC, La Philosophie de la nature. Première partie: la nature en général, fasc. 2-3, en Angelicum 36 (1959) 456; 37 (1960) 113-114.

Recensión sin firma sobre J. DE TONQUÉDEC, La Philosophie de la nature. Première partie: la nature en général, fasc. 2-3, en Gregorianum 41 (1960) 119.

Recensión sin firma sobre J. DE TONQUÉDEC, La Philosophie de la nature. Première partie: la nature en général, fasc. 2-3, en Revue de Sciences Religieuses 35 (1961) 99.

Recensión sin firma sobre J. DE TONQUÉDEC, La Philosophie de la nature. Première partie: la nature en général, fasc. 2-3, en Giornale de Metafisica 16 (1961) 770-771.

Recensión sin firma sobre J. DE TONQUÉDEC, La critique de la connaissance ( $3^{\mathrm{a}}$ ed.), en La Ciencia Tomista 88 (1961) 280; 649-650.

Recensión sin firma sobre J. DE TONQUÉDEC, La critique de la connaissance ( $3^{\mathrm{a}}$ ed.), en Ciencia y Fe 17 (1961) 343-344.

Recensión sin firma sobre J. DE TONQUÉDEC, La critique de la connaissance ( $3^{\mathrm{a}}$ ed.), en Philosophical Studies 11 (1961-62) 303.

Recensión sin firma sobre J. DE TONQUÉDEC, La critique de la connaissance ( $3^{\mathrm{a}}$ ed.), en Revue d'Histoire et Philosophie religieuses 42 (1962) 60-62. 
Recensión sin firma sobre J. DE TONQUÉDEC, La critique de la connaissance $\left(3^{\mathrm{a}}\right.$ ed.), en Bijdragen. Tijdscrift voor philosophie en theologie 23 (1962) 97.

Recensión sin firma sobre J. DE TONQUÉDEC, La critique de la connaissance ( $3^{\mathrm{a}}$ ed.), en Estudios Filosóficos 11 (1962) 333334.

Recensión sin firma sobre J. DE TONQUÉDEC, La critique de la connaissance ( $3^{\mathrm{a}}$ ed.), en Études franciscaines 12 (1962) 239240.

Recensión sin firma sobre J. DE TONQUÉDEC, La critique de la connaissance ( $3^{a}$ ed.), en L'Ami du Clergé 72 (1962) 153-154.

Recensión sin firma sobre J. DE TONQUÉDEC, La critique de la connaissance ( $3^{\mathrm{a}} \mathrm{ed}$.), en Revue Thomiste 70 (1962) 168-169.

Recensión sin firma sobre J. DE TONQUÉDEC, La Philosophie de la nature. Première partie: la nature en général, fasc. 2-3, en Pensamiento 18 (1962) 231-232.

Recensión sin firma sobre J. DE TONQUÉDEC, La Philosophie de la nature. Première partie: la nature en général, 'fasc. 2-3, en Bulletin Thomiste 12 (1960-62) 142-143.

Recensión sin firma sobre J. DE TONQUÉDEC, La Philosophie de la nature. Deuxième partie: la nature vivante et connaissante, en Bulletin Thomiste 12 (1962-63) 304.

Recensión sin firma sobre J. DE TONQUÉDEC, La Philosophie de la nature. Deuxième partie: la nature vivante et connaissante, en Nova et Vetera 38 (1963) 151.

Recensión sin firma sobre J. DE TONQUÉDEC, La Philosophie de la nature. Deuxième partie: la nature vivante et connaissante, en The Downside Review 81 (1963) 289-290.

Recensión sin firma sobre J. DE TONQUUÉDEC, La Philosophie de la nature. Deuxième partie: la nature vivante et connaissante, en L'Ami du Clergé 73 (1963) 479.

Recensión sin firma sobre J. DE TONQUÉDEC, La Philosophie de la nature. Deuxième partie: la nature vivante et connaissante, en Studium 3 (1963) 619-620. 
Recensión sin firma sobre J. DE TONQUÉDEC, La Philosophie de la nature. Deuxieme partie: la nature vivante et connaissante, en Ciencia y Fe 19 (1963) 79.

Recensión sin firma sobre J. DE TONQUÉDEC, La Philosophie de la nature. Deuxième partie: la nature vivante et connaissante, en La Ciencia Tomista 90 (1963) 526-528.

Recensión sin firma sobre J. DE TONQUÉDEC, La Philosophie de la nature. Deuxième partie: la nature vivante et connaissante, en Études Philosophiques 18 (1963) 482-483.

Recensión sin firma sobre J. DE TONQUÉDEC, La Philosophie de la nature. Deuxième partie: la nature vivante et connaissante, en Divus Thomas 66 (1963) 497.

Recensión sin firma sobre J. DE TONQUÉDEC, La critique de la connaissance ( $3^{\mathrm{a}}$ ed.), en Scholastik 38 (1963) 125.

Recensión sin firma sobre J. DE TONQUÉDEC, La critique de la connaissance $\left(3^{\mathrm{a}}\right.$ ed.), en Bulletin de Littérature Ecclésiastique 64 (1963) 233.

Recensión sin firma sobre J. DE TONQUÉDEC, La Philosophie de la nature. Deuxième partie: la nature vivante et connaissante, en Zeitschrift für katholische Theologie 86 (1964) 109.

Recensión sin firma sobre J. DE TONQUÉDEC, La Philosophie de la nature. Deuxième partie: la nature vivante et connaissante, en Angelicum 41 (1964) 118-119.

Recensión sin firma sobre J. DE TONQUÉDEC, La Philosophie de la nature. Deuxième partie: la nature vivante et connaissante. en Revue Philosophique 62 (1964) 519-520.

Recensión sin firma sobre J. DE TONQUÉDEC, La Philosophie de la nature. Deuxième partie: la nature vivante et connaissante, en Revue des Sciences philosophiques et théologiques 49 (1965) 114. 
e. Sobre las otras obras de Tonquédec

G. DELANNOYE, S.J., recensión de: J. DE TONQUÉDEC, Sur la Philosophie bergsonnienne, en Nouvelle Revue Théologique 6 (1938) 753.

A. GRÉGOIRE, S.J., recensión de: J. DE TONQUÉDEC, Les maladies nerveuses ou mentales et les manifestations diaboliques, en Nouvelle Revue Théologique 2 (1939) 238.

Recensión sin firma sobre J. DE TONQUÉDEC, Les maladies nerveuses ou mentales et les manifestations diaboliques, en Revue thomiste 45 (1939) 203.

R.T., recensión de: J. DE TONQUÉDEC, Une Philosophie existentielle. L'existence d'après Karl. Jaspers, en . Nouvelle Revue Théologique 10 (1949) 11.15. 
Copyright of Tópicos. Revista de Filosofía is the property of Universidad Panamericana and its content may not be copied or emailed to multiple sites or posted to a listserv without the copyright holder's express written permission. However, users may print, download, or email articles for individual use. 